\title{
Los espacios libres públicos como expresión de la identidad de los barrios centrales de Recife
}

\author{
Sandra Augusta Leão Barros
}

Filiación: Arquitecta y Urbanista (UFPE), Master y Doctoranda en Estructuras Ambientales Urbanas (FAU.USP). E-mail: sandleao@gmail.com

\section{NDI CE}

\author{
I ntroducción \\ El trazado histórico-geográfico de los barrios centrales \\ La disposición espacial de los espacios libres públicos \\ La identidad contemporánea del centro - la 'cara' actual \\ Conclusión \\ Referencias bibliográficas
}

\section{RESUMEN}

Recife, al racif, origen de su nombre - baixio o acera de piedra, en homenaje a la barrera de arrecifes naturales existentes, es el nombre de la ciudad que tuvo en la plástica natural su función primaria - núcleo portuario, y tiene hasta hoy su identidad ligada a los espacios libres de sus barrios centrales, su forma natural da lugar a la forma construida, siendo moldeada por esta ${ }^{1}$. Como porción de tierra tomada en parte al río, y en parte al mar, aspira desde siempre a la libertad, destacando su espíritu colectivo, y el gusto por los horizontes libres permitiendo observar el gran océano y los ríos que la rodean. Desde el dominio holandés (1630-1654), período que vio una gran reforma urbana y figuración de su centro (formado por varias islas y puentes), hubo preocupación por presencia de espacios libres diversos en su trazado: jardines del palacio, márgenes de muelles y cabezas de puentes, patios de iglesias e/o mercados. El trazado urbano de los barrios centrales -Barrio del Recife, Santo Antonio y San José, empieza a tomar cuerpo y significado tomándose en cuenta también la anchura de las vías y gabarito de las manzanas, perfil que continua hasta hoy en gran parte de esos barrios, aunque las fachadas hayan sido cambiadas ${ }^{2}$.

PALABRAS-CLAVE: ESPACIOS LIBRES PÚBLICOS, BARRIOS, CENTRO DE LA CIUDAD, IDENTIDAD URBANA, RECIFE.

\section{ABSTRACT}

Recife, al racif, its original name - slow stone sidewalk, in tribute of the natural reef barrier which indeed exists, city that had in the natural plastic form its primary

\footnotetext{
1 'La naturaleza construyendo los arrecifes a lo largo de la costa generó el muelle natural. El hombre levantando en las tierras bajas, situadas por detrás de esos arrecifes, el organismo artificial de sus construcciones, dio lugar la ciudad. De la asociación de esos dos factores geográficos - la utilización del espacio como si fuera una sustancia plástica a ser modelada-, surgió el paisaje natural de esa ciudadpuerto". CASTRO, Josué de. Un ensayo de Geografía Urbana: la Ciudad del Recife. En: Ensaios de Geografia Humana. São Paulo: Brasiliense, 1957, p. 280.

${ }^{2}$ Conforme estudios del historiador José Luiz da Mota Menezes, que se viene dedicando a este asunto en particular y es autor del proyecto de reforma de la sinagoga de la calle Buen Jesús - Barrio del Recife.
} 
function - harbour center; has until nowadays your identity connected into its open spaces of your central districts, your natural shape gives place to your constructed shape, being moulded by itself ${ }^{3}$. Earth portion took half by the rivers, half by the sea, aspirate since from to liberty, to collective spirit, to open horizons where anyone could observe the ocean and the rivers which surround it. Since the Dutch period (16301654), period when was a large urban reform and city center pattern (which is formed by some islands and bridges), there was preoccupation into the presence of open public spaces in its pattern, which could be palace's gardens, quaysides, head bridges, church yards and/or market. The urban design of its central districts - Recife's district, Santo Antonio and Sao Jose, begins to have body and meaning taking into account also the streets width and quartier's height, outline which stands until now in most part of these districts, although its façades have been changed ${ }^{4}$.

KEY-WORDS: OPEN PUBLIC SPACES, DISTRICTS, CITY CENTER, IDENTITY, RECIFE

\section{I ntroducción}

Los versos de un historiador y gran conocedor de los problemas urbanos - "Es de los sueños de los hombres que una ciudad se inventa"-, nos traen la idea del espacio público y el vivir colectivo como algo siempre deseado, siempre presente y prioritario en Recife.

Al pasar de los años y de las reformas urbanas que tiene su centro, en Recife las campanas de sus iglesias continúan puntuando el paisaje central, los rituales católicos de misas y cortejos marcan aún el calendario anual de las festividades, y los grupos de carnaval salen y llegan de los patios de las iglesias; de igual forma, los ríos continúan bañando el agitado centro.

Los patios de las iglesias congregan a las personas, las plazas atraen visitantes, las flanelinhas y pidientes también los hacen su territorio, lo que no es diferente de otras ciudades brasileras.

Lo que se propone este artículo es detener la mirada sobre la morfología de esos espacios en la malla urbana recifense para ver cómo hasta hoy representan los puntos identificativos de esos barrios, junto a los puentes y orillas de río.

\section{El trazado histórico-geográfico de los barrios centrales}

El centro de Recife está compuesto por varias partes, sean ellas sólidas o líquidas. Cuando se dice centro, el recifense no tiene unA imagen de un núcleo único, sino de varios pedazos que componen un todo más amplio.

Al inicio hubo una planicie entre el agua del mar y de los ríos, una planicie minúscula con pequeños almacenes, comerciantes, tabernas, servicios conectados al muelle (el que corresponde hoy al Barrio del Recife). La Ciudad Mauricia se expande hacia el

\footnotetext{
3 "The nature building the stone sidewalks along the coast built the natural harbour. Man building at low lands, situated behind of these stone sidewalks, the artificial organism of those buildings, built the city. By the association of the two geographic factors - the space using as being a plastic substance to be built, borned the natural landscape of this harbour-city". CASTRO, Josué de. An essay of Urban Geography: the City fo Recife. In: Essays of Human Geography. São Paulo: Brasiliense, 1957, p. 280.

${ }^{4}$ According to researches of the historian Jose Luiz da Mota Menezes, which has been studying this theme in particular, and is author of the project of reform of the synagogue situated at Bom Jesus street - Recife's district.
} 
interior de la planicie dando origen a la nueva Mauricia, llamada así en el período holandés, hoy barrios de Santo Antonio y San José. En sucesivos terraplenes y expansiones, la ciudad continúa y se propaga para la Boa Vista; posteriormente, con la ocupación de los judíos próxima al Muelle José Mariano, va adoptando una nueva forma de vivir en el interior de sus manzanas.

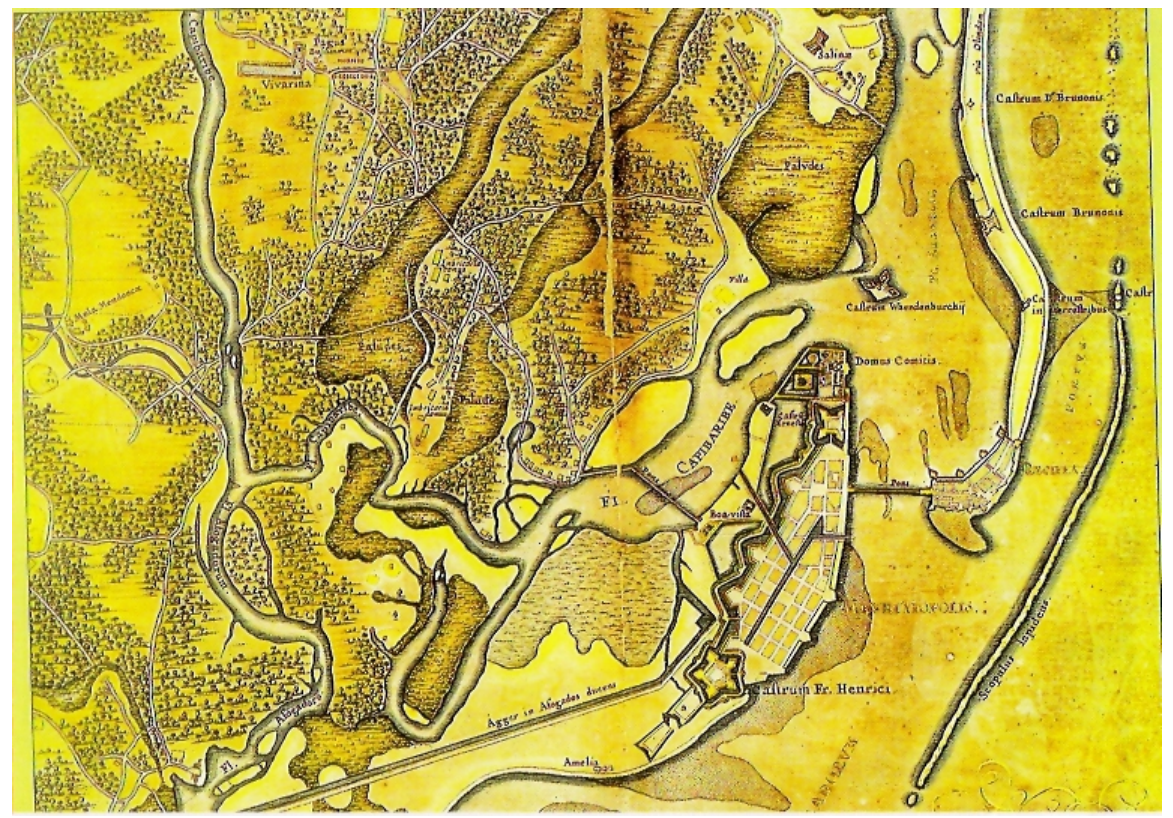

Fig. 1.- Trazado central en el período holandés. Imagen extraída del libro de Gaspar Barleus, 1647. En: HERKENHOFF, Paulo (org.). EL Brasil y los holandeses: 1630-1654. Rio de Janeiro: Sextante, 1999, p. 93, ISBN 8586796-26-3.

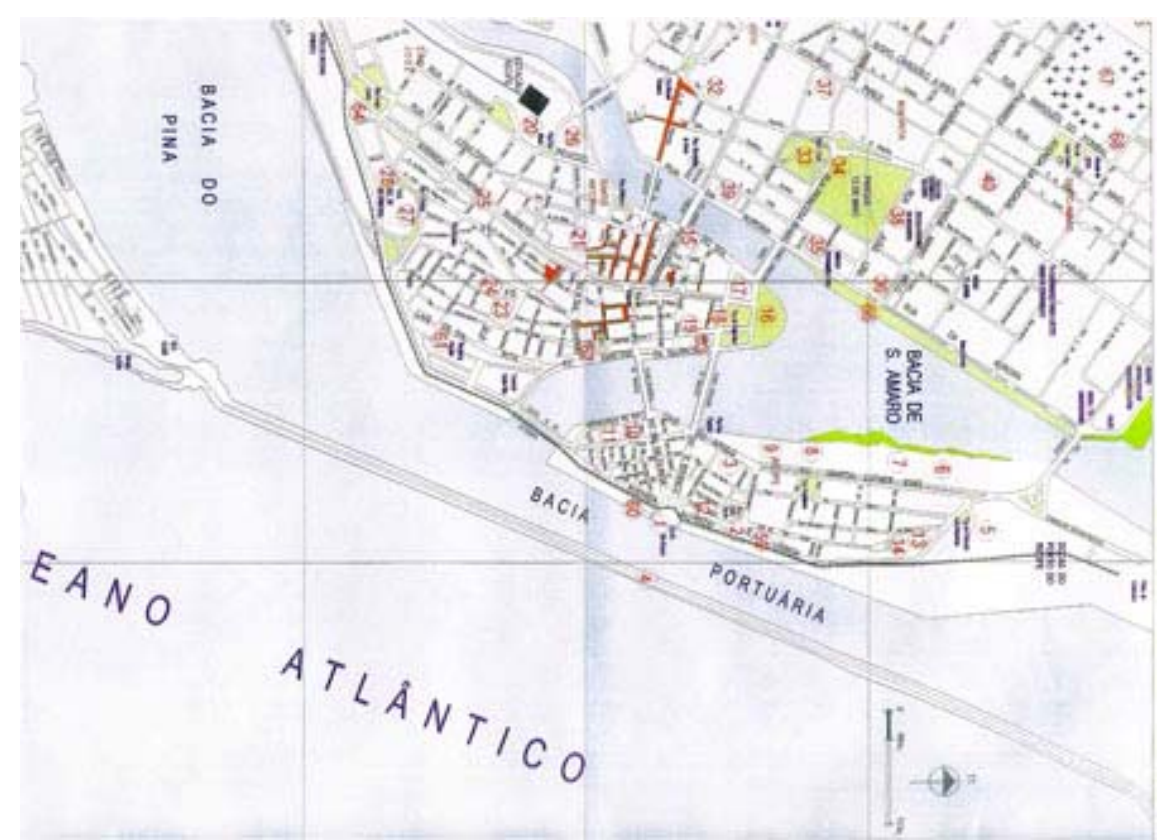

Fig. 2.- Trazado central en la actualidad. Carta Planimétrica de Recife, $\mathrm{n}^{\circ}$ 90-05. Recife: Fidem, 2000, esc. 1:10.000. 


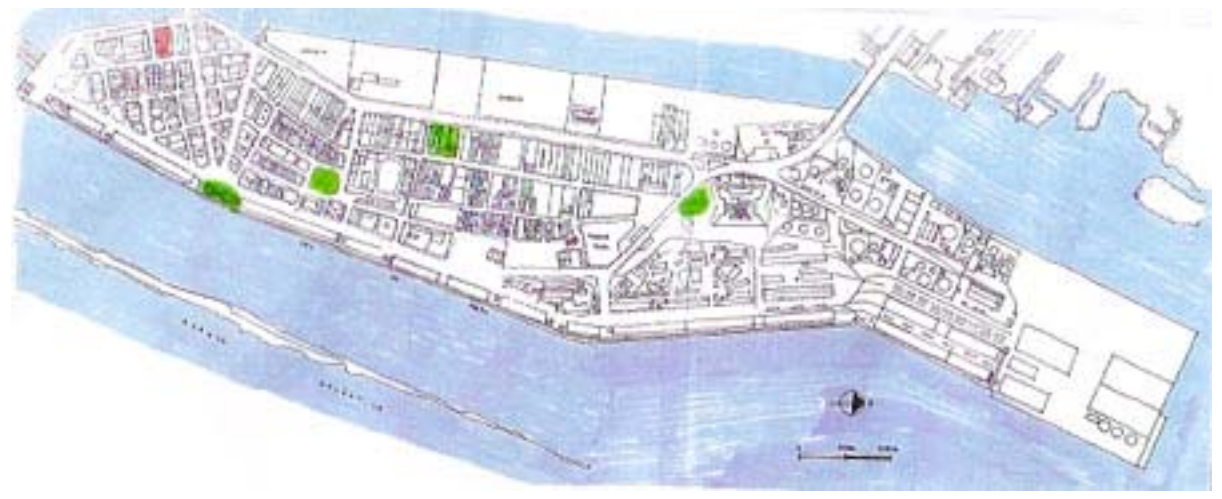

Fig. 3.- Trazado del Barrio del Recife. Unibases $n^{\text {os }} 91 / 40: 00 ; 90 / 49: 05 ; 90 / 49: 00$; 90/48:05. Recife: Fidem, 1990, esc. 1:1000.

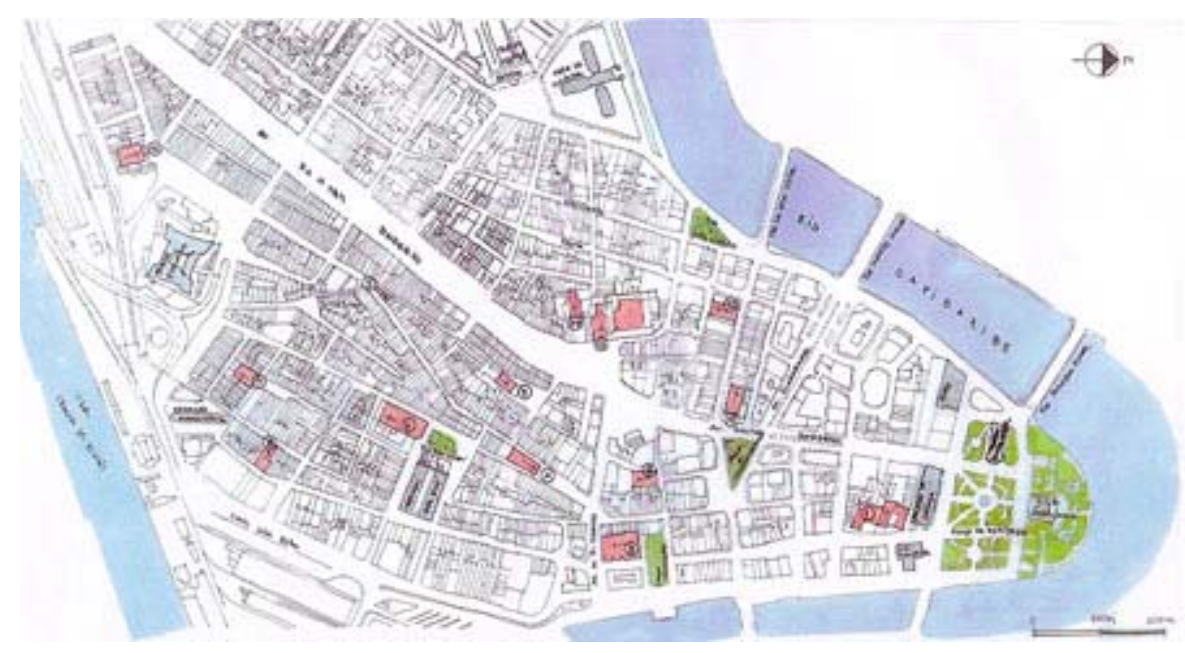

Fig. 4.- Trazado de los barrios de Santo Antonio y San José. Unibases nos $91 / 30: 00$; 90/39:05; 90/39:00； 90/38:05; 90/38:00; 90/37:05; 90/37:00; 90/28:05; 90/28:00; 90/27:05; 90/27:00; 90/18:00. Recife: Fidem, 1990, esc. 1:1000.

\section{La disposición espacial de los espacios libres públicos}

Buscándose una forma de clasificación para poder comparar, se llega a los tipos referidos abajo:

a) Patios de forma cuadrangular dispuestos frontalmente al templo 


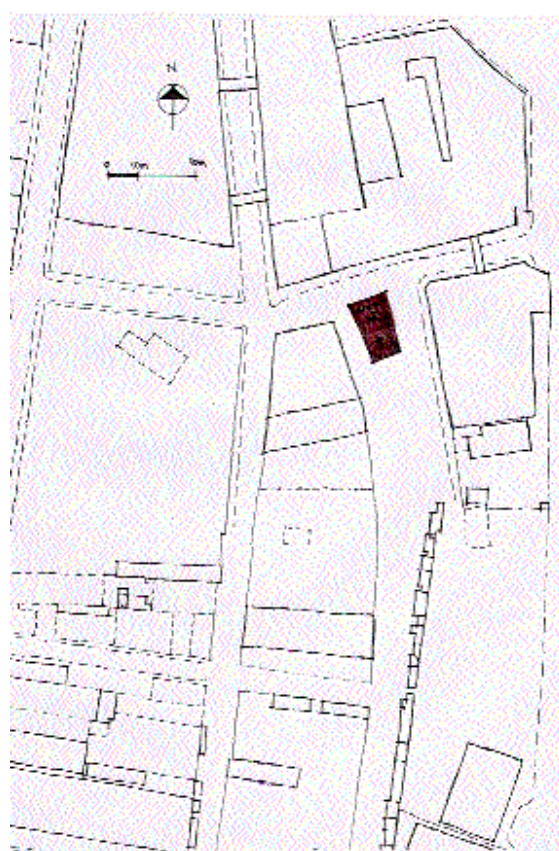

Fig. 5.- Iglesia del Pilar - Barrio del Recife

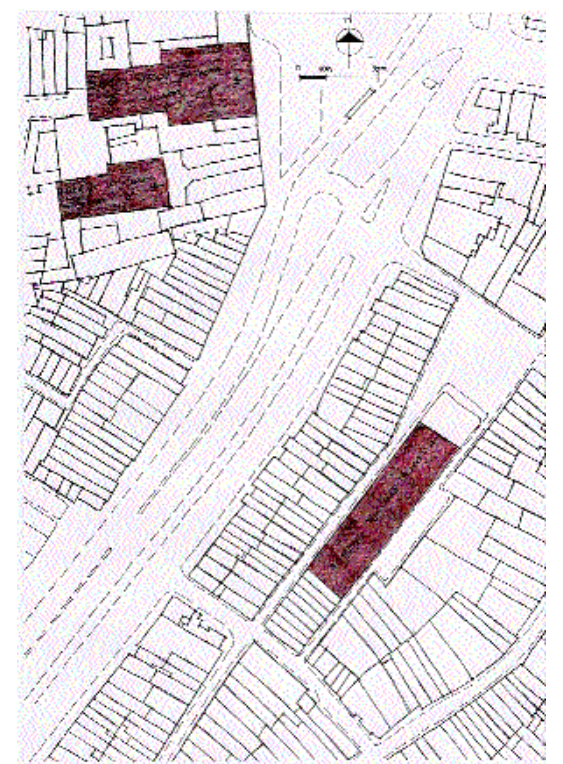

Fig. 6.- Iglesia de San Pedro (en el cuadrante inferior derecho de la imagen) 
b) Patios en forma de ' $V$ ', teniendo el templo en su ápice

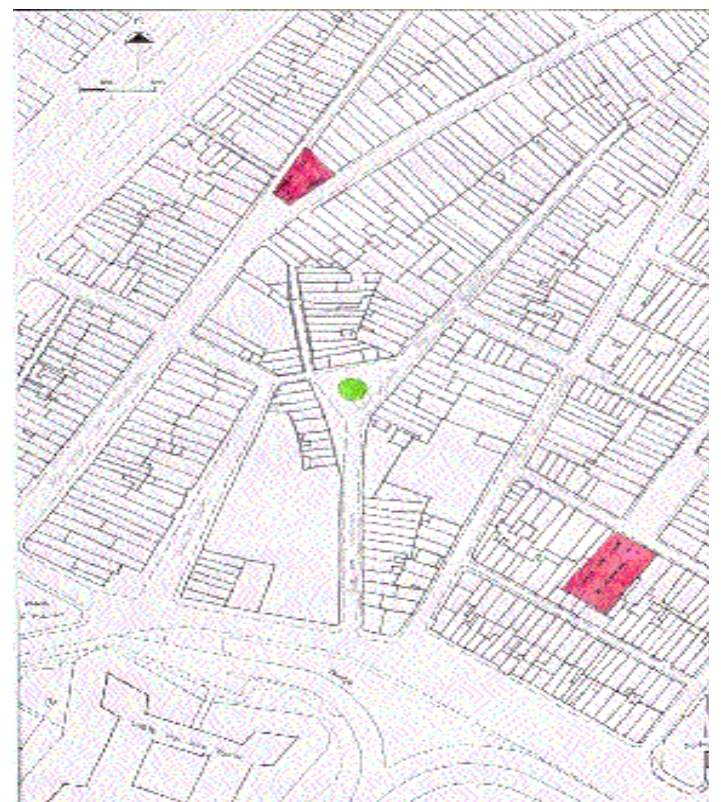

Fig. 7.- Iglesia de Nuestra Sra. del Tercio (iz.) e Iglesia de San José de Ribamar (der.), plaza en calle del Jardín y Padre Floriano; plaza "Forte das Cinco Pontas".

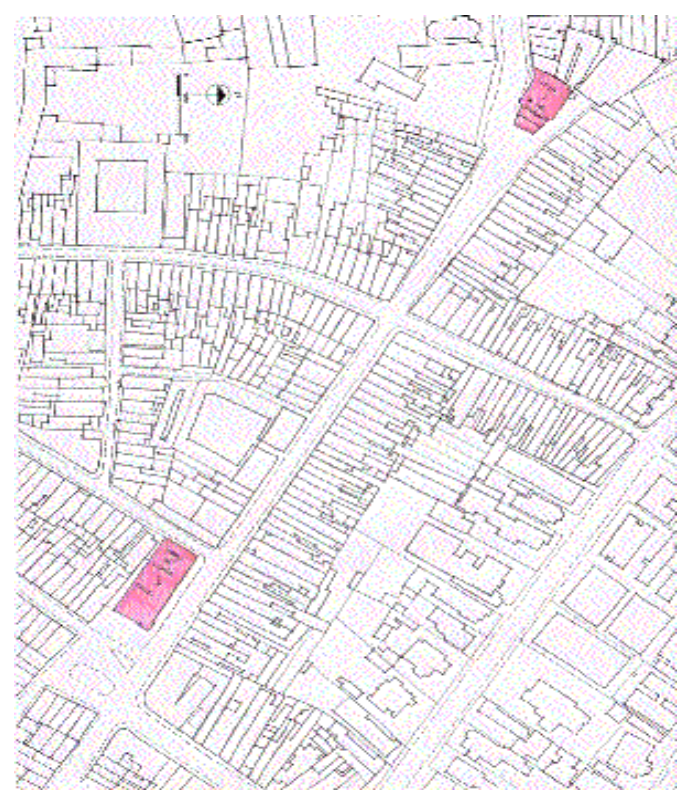

Fig.8.- Iglesia de San Gonzalo e Iglesia de Santa Cruz, Plaza en la Calle del Jardín 
c) Plaza lateral $y / 0$ frontal al templo, con o sin mercado

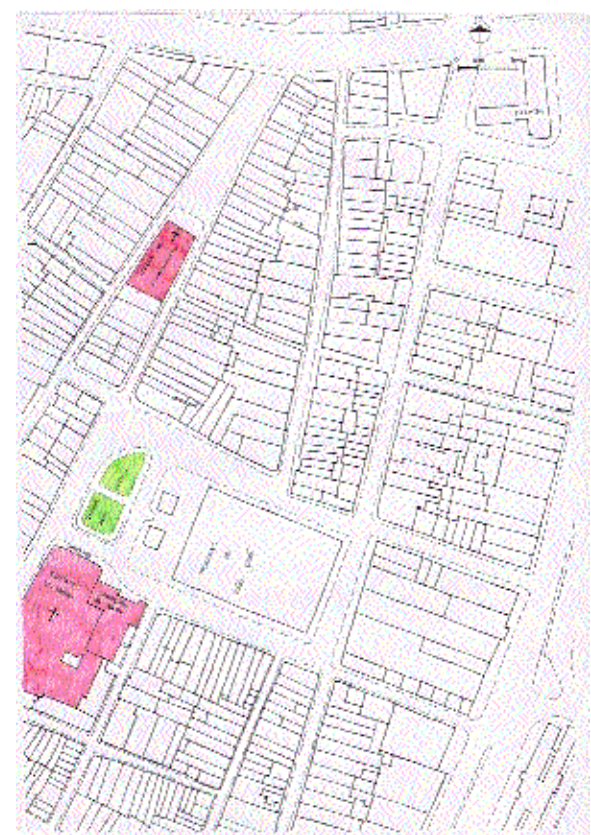

Fig.9.- Nuestra Señora de la Penha Basílica y convento) + Plaza Don Vital + Iglesia Mercado de San José, iglesia del Libramiento

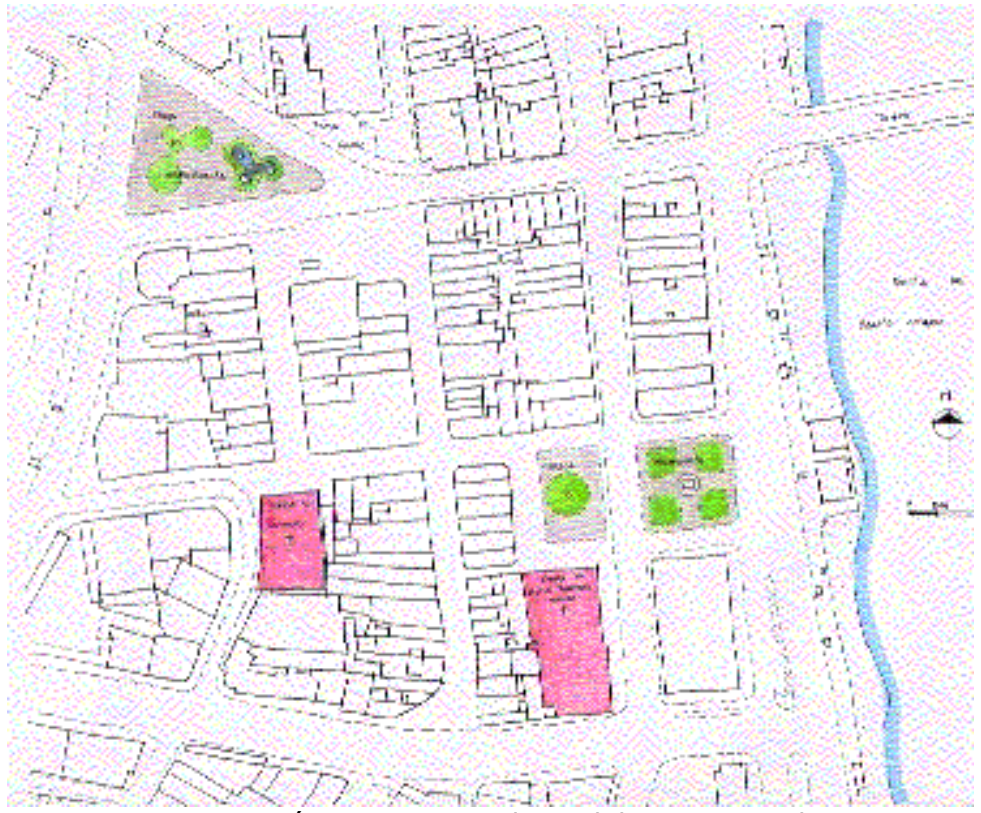

Fig. 10.- Divino Espíritu Santo + Iglesia del Rosario + Plaza Diecisiete, (próximas las plaza de la Independencia y de Diario). 
d) Plaza en el interior de la cuadra

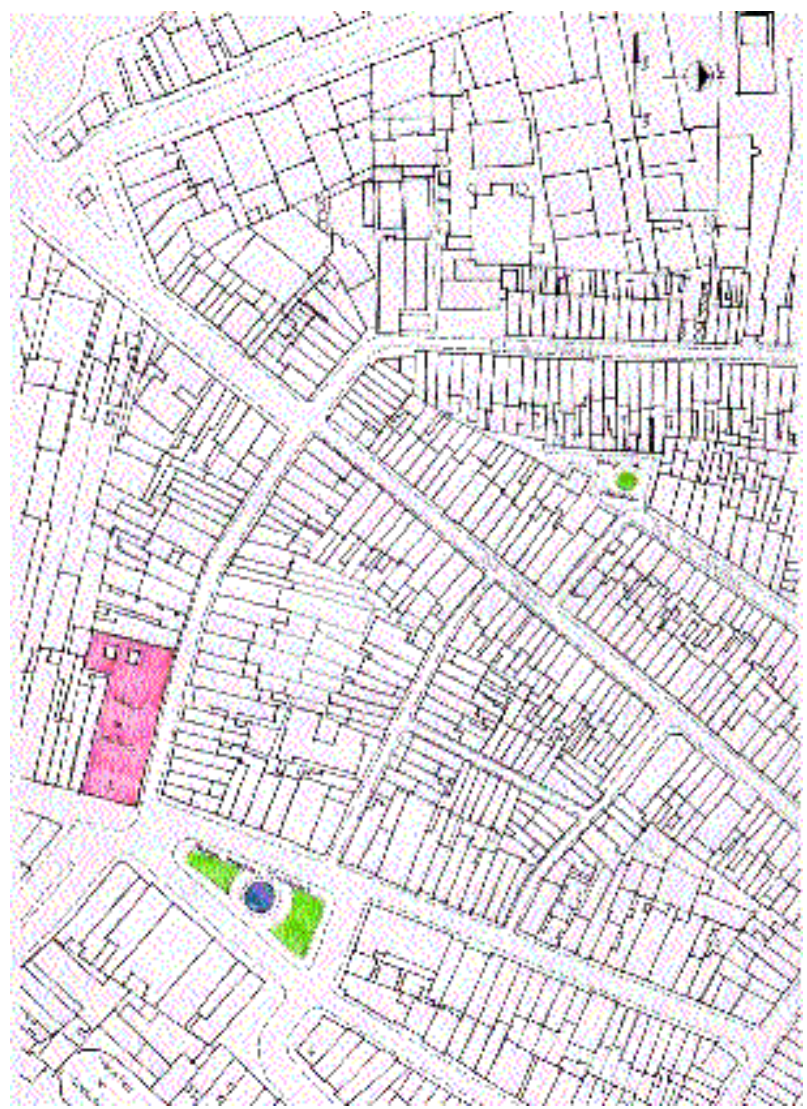

Fig. 11.- Iglesia de la Matriz y plaza de Marcial Pinheiro más arriba Plaza de la Alegría - Boa Vista.

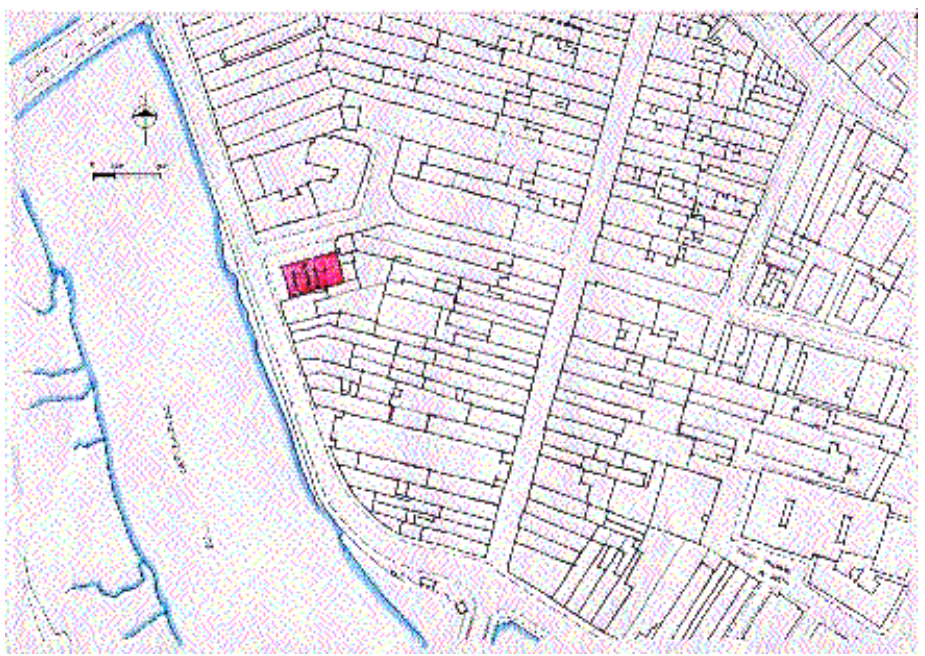

Fig. 12.- Iglesia Presbiteriana y Plaza Machado de Assis (detrás del cine San Luis, en el cuadrante inferior derecho de la imagen) 


\section{La identidad contemporánea del centro - la 'cara' actual}

La identificación propuesta facilita la lectura de esos espacios, pero la malla urbana es compleja y transciende mucho tal clasificación, es preciso que se esté atento a esto.

Los edificios contiguos a estos espacios son, en gran parte de las situaciones, similares en lineamientos frontales, mirando a la calle.

El paisaje que deriva hace un efecto también homogéneo: la evocación de la grandiosidad del templo a través de los espacios contiguos frontales o laterales, o el remanso de las placitas en el interior de la manzana se hace sentir en un ejemplo o en otro. Son dos formas de apropiación del espacio urbano, dos escalas urbanas concomitantes. Ambas trabajan la escala del pedestre, conforme a la época de sus construcciones, cuando el transporte urbano estaba asociado al caballo.

\section{Conclusión}

Los patios de iglesias son comunes en varias otras ciudades brasileras de la misma época; la convivencia con el agua se traduce en una particularidad de la ciudad de Recife. Los barrios centrales dan cuenta de una especificidad contribuyente a su identidad y percepción.

Si bien es cierto que este es el primer texto en que se empieza a trabajar tales espacios como parte integrante de un estudio más amplio que merece más profundización y madurez, ya se puede identificar sus principales aspectos.

Poder comparar tales espacios con ejemplos de otras mallas urbanas brasileras y también de origen hispánico puede generar contribuciones valiosas que develen el por qué de su éxito y permanencia a pesar del paso del tiempo.

\section{Referencias bibliográficas}

ALMEIDA, Érika Audet de. La articulación de los espacios públicos en el Recife del siglo XIX. Recife: Disertación de Master (MDU.UFPE), 2001.

BARTALINI, Vladimir. Los espacios libres públicos como expresiones de centralidad. En: Paisaje Ambiente: ensayos, San Paulo. N. 3, p. 07-14, 1989. ISSN 0104/6098.

BRAGA, João. Trillas del Recife: guía turístico, histórico y cultural. Recife: Bagaço, 2007. ISBN 978-85-373-0299-6.

COSTA, Pereira da. Alrededores del Recife. Recife: Fundaj/Massangana, 2001. ISBN 85-7019-381-5.

CASTRO, Josué de. Un ensayo de Geografía Urbana: la Ciudad de Recife. En: Ensayos de Geografía Humana. San Paulo: Brasiliense, 1957, p. 193-280.

GUERRA, Flavio. Viejas iglesias y subúrbios históricos. Recife: Ayuntamiento Municipal del Recife, 1978.

LEITE, Rogério Proença. Contra-usos de la ciudad: lugares y espacio público em la experiencia urbana contemporánea. Campinas: Editora de la Unicamp, Aracaju: Editora UFS, 2004. ISBN 85-268-0681-5.

LUDEMIR, Rosa Bernarda. Un lugar judío en el Recife: la influencia de elementos culturales em el proceso de apropiación del espacio urbano del barrio de Boa Vista por la imigración judía en la primera mitad del siglo XX. Recife: Disertación de Master (MDU.UFPE), 2005.

MARX, Murillo. Ciudad en Brasil: tierra de quien? San Paulo: Edusp, 1980. ISBN 85213-0650-4. 
Planta Cadastral nos 90/18:00, 90/28:05, 90/28:05, 90/27:05, 90/27:00, 90/30:00, 90/39:05, 90/39:00, 90/38:05, 90/38:00, 90/37:05, 90/37:00, 91/40:00, 90/49:05, 90/49:00, 90/48:05. Recife: Fidem, 1989.

RECIFE, Ayuntamiento de la Ciudad del. Preservación de sitios históricos. Recife: Empresa de Urbanización, 1981.

REIS FILHO, Nestor Goulart. Contribución al estudio de la evolución urbana del Brasil: 1500-1720. San Paulo: Pini, 2000. ISBN 85-7266-116-6.

SÁ CARNEIRO, Ana Rita, MESQUITA, Liana de Barros (orgs.). Espacios libres del Recife. Recife: Ayuntamiento Municipal/ UFPE, 2000.

TEIXEIRA, Manuel C. (org.). La plaza en la ciudad portuguesa. Lisboa: Libros Horizonte, 2001. ISBN 972-24-1120-9. 